\title{
Leaky Waveguide for Train-to-Wayside Communication-Based Train Control
}

\author{
Marc Heddebaut, Member, IEEE
}

\begin{abstract}
In guided urban automated transportation systems, the maintenance of a high-quality broadband train-to-wayside communication at all locations along the track poses a major problem. Several techniques are currently used. However, to achieve sufficient rates, a communication frequency range of $2-6 \mathrm{GHz}$ is widely in use. At these frequencies, the natural propagation in tunnels usually works efficiently, but the communication through a leaky waveguide that is continuously laid along the track has several advantages. Over the required communication ranges of several hundred meters, between stations, such waveguides provide low longitudinal attenuation and remain of reasonable size. They also provide predictable communication ranges that could offer significant advantages over the natural propagation approach. This paper will theoretically and experimentally describe such a technical solution. Using ray-optical modeling of the radio propagation in a railway-type tunnel, the radiation characteristics of such a system will be evaluated. At the same operating frequencies, it will be compared with a conventional base solution using antennas separated a few hundred meters apart. An example of a recently operational implemented system will be presented.
\end{abstract}

Index Terms - Leaky wave antennas, propagation, train control, tunnels, waveguides.

\section{INTRODUCTION}

$\mathbf{F}$ OR THE control and command of railways and underground tunnels, the control centers must receive information from all the vehicles in the system. Among other vital items of information, one can mention localization, speed, acceleration, and the identity of the vehicles. This track to train the data exchange is often denominated by the acronym CBTC, which stands for communication-based train control. To improve the efficiency and economy of an automated transportation system, a major requirement is thus to maintain a reliable and broadband communication link between the vehicles and the ground computers. Moreover, for several years, we have noticed a large increase in the volume of data exchanged to secure Automatic Train Control (ATC), Automatic Train Operation (ATO), and Automated Train Protection (ATP).

Because it is difficult to develop surface corridor transport in crowded urban environments, urban-guided transportation systems are often deployed in underground tunnels. Thus, for these systems, it is vital to have an effective and highly

Manuscript received July 12, 2007; revised February 26, 2008, May 13, 2008, and June 20, 2008. First published July 16, 2008; current version published March 17, 2009. The review of this paper was coordinated by Prof. Z. Yun.

The author is with the French National Institute for Transport and Safety Research (INRETS), F-69675 Bron Cedex, France (e-mail: marc. heddebaut@inrets.fr).

Color versions of one or more of the figures in this paper are available online at http://ieeexplore.iee.org.

Digital Object Identifier 10.1109/TVT.2008.928635 available track-to-train tunnel operating communication system [1], [2]. The current requirements for CBTC, particularly for automated subway systems, necessitate significantly high data rates, which are typically several megabits per second. Communication ranges of several hundred meters, between stations, are also required. To achieve these transmission rates, the current technical solutions use radio modems working at high carrier frequencies (between 2 and $6 \mathrm{GHz}$ ). For some industrial suppliers, the radio modems are developed, with some adjustments, to comply with the existing wireless local area network (WLAN) standards, i.e., IEEE $802.11 \mathrm{~g}$ or a, but they could evolve, in the future, to the IEEE $802.11 \mathrm{p}$ standard. Some other industrial suppliers have developed proprietary scheme CBTC radio systems that also work in the same frequency range. IEEE is performing a standardization work on CBTC, and IEEE RTVISC-1473 considers the different train-wayside communication solutions provided by train control suppliers.

\section{STUdied Systems}

These CBTC and associated radio modems have to work in the specific tunnel propagation channel. On the ground side, these modems are either connected to antennas a few hundred meters apart, for example, 400-600 m along the tunnel, or alternatively connected to continuous radiating structures. At frequencies below $1 \mathrm{GHz}$, leaky coaxial cables are often used as a continuous radiating structure. To obtain the necessary coupling and radiation of energy outside of the coaxial structure, different slot shapes have been used [3]-[6]. Leaky coaxial cables operated at 450 or $800 \mathrm{MHz}$ are widely used in railways [7]. However, at frequencies above $1 \mathrm{GHz}$, the dielectric losses inside coaxial cables become very significant. This leads to high longitudinal attenuation values. At $2.5 \mathrm{GHz}$, a $42-\mathrm{mm}$-diameter cable attenuation is on the order of $80 \mathrm{~dB} / \mathrm{km}$. To cope with this problem, one technique is to suppress the cable dielectric material and the inner conductor, thus using an empty waveguide. Between 2 and $6 \mathrm{GHz}$, a waveguide operating in the fundamental mode remains of reasonable size and provides a low longitudinal attenuation, which is adequate for our selected ranges of several hundred meters. Such a robust structure provides a transmission line that is electrically and mechanically compatible with the difficult track environment. Working in the fundamental mode also leads to a propagation line that is capable of handling subway track curvatures without the need for waveguide mode-filtering equipment. For ranges of 20-30 km, very long range applications, higher waveguide propagation modes, and associated mode-filtering devices were used by Kawakami et al. [8]. This paper will theoretically 
and experimentally develop such a solution. At $2.4 \mathrm{GHz}$, the leaky waveguide tunnel radiation characteristics are evaluated and compared with a base solution using conventional antennas operated in the same tunnel environment.

This paper is organized as follows. Section III briefly presents the tunnel propagation channel that is simulated using ray-optical modeling of the radio propagation. Section IV presents the results obtained using one and then two consecutive antennas situated at realistic locations inside the tunnel. This will be used as a base technique. Section V models the leaky waveguide intrinsic radiation and its radiation inside the tunnel. Section VI provides the simulation results of the inthe-tunnel leaky waveguide radiation and then of a mixed antenna plus leaky waveguide configuration. Section VII presents an example of a recently implemented system. Section VIII gives the experimental results of the leaky waveguide radiation in a tunnel and deduces some conclusions.

\section{Tunnel Propagation Channel}

Depending on the local regulation, as well as on the industrial solution chosen by the transport operator, the current CBTC systems are operated in the 2- to 6-GHz frequency range. Since in this frequency range the wavelength is much smaller than the transversal dimensions of urban transportation tunnels, tunnel propagation can be considered as in an oversized waveguide. This condition makes it possible to apply the asymptotic geometrical optics theory. Then, the tunnel can be modeled as a multipath channel with white Gaussian noise. In that case, we can represent it by the time variant channel given by

$$
h(t, \tau)=\sum_{k=0}^{N(t)-1} a_{k}(t) \delta\left[\tau-\tau_{k}(t)\right] e^{j \theta_{k}(t)}
$$

where $a_{k}$ is the $k$ th path amplitude value, $\tau_{k}$ is the arrival time of the $k$ th path, $\theta_{k}$ is the $k$ th path phase value, $\delta$ is the Dirac delta function, and $N$ is the number of resolvable multipaths. If the channel is supposed to be stable during the relevant periods, the time invariant version can be used. It is then expressed as

$$
h(\tau)=\sum_{k=0}^{N-1} a_{k} \delta\left(\tau-\tau_{k}\right) e^{j \theta_{k}} .
$$

To study tunnel propagation, deterministic approaches offer high accuracy and can provide information about significant parameters such as the small-scale fading or the delay dispersion. Among the deterministic approaches, ray-tracing methods firstly consist of determining the ray trajectory from the transmitter to the receiver, and secondly, once this trajectory is available, determining the actual field strength conveyed by any ray reaching the receiver. In a given geometry, the determination of the rays' trajectories leaving the transmitter toward the receiver can be made in a direct or indirect way. The direct method, using, for example, the image theory, leads to an exact ray trajectory. For the indirect method, which is often called ray launching, a number of rays are launched from the transmitter to the receiver in arbitrary directions using a known angular step. Didascalou $e t$ al. obtained good results using a small angular step [9], [10]. In the early 1970s, Mahmoud and Wait derived

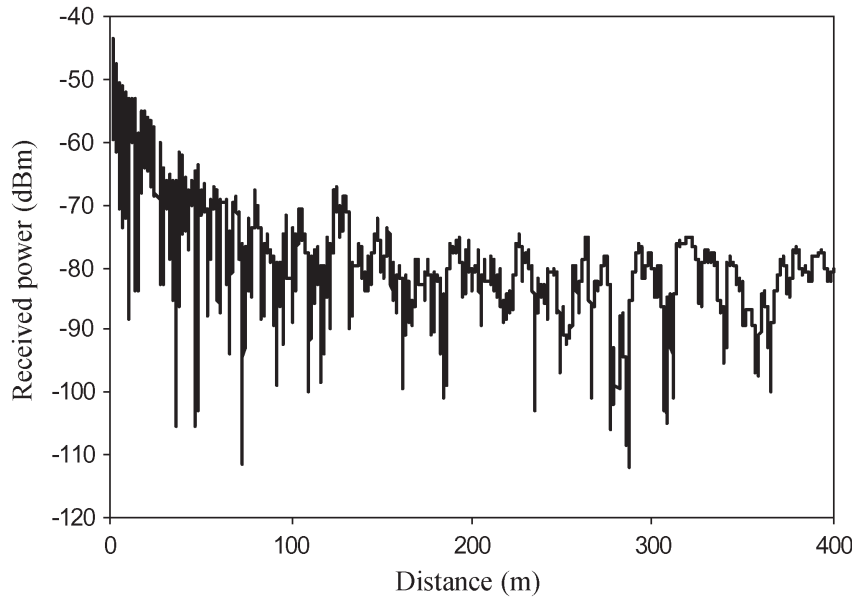

Fig. 1. Simulated first AP located $0 \mathrm{~m}$ along the track.

a geometrical ray model for straight rectangular tunnels [11]. Starting from this model, Zhang and Hong explored simulcast radio propagation in tunnels at $900 \mathrm{MHz}$ and $2 \mathrm{GHz}$ [12]. Zhang and Hwang developed the theory of the radio wave in railway tunnels in [13]. A detailed description of the straight rectangular tunnel model using the ray-tracing technique based on geometrical optics is provided in [12]. We use the same model to establish our subway tunnel antenna radio coverage at $2.4 \mathrm{GHz}$.

\section{Base Radio Coverage Using Antennas}

Using this model, simulations were performed using an 8-m-wide and 6-m-high infinite empty rectangular-shaped parallel-track subway-type tunnel. For the tunnel walls, a dielectric constant $\varepsilon_{r}=10$ and a conductivity $\sigma=0.01 \mathrm{~S} / \mathrm{m}$ were selected [12]. The antennas are situated at realistic track locations (width, height), i.e., $x_{0}=7 \mathrm{~m}, y_{0}=5.5 \mathrm{~m}$ for the emitter and $x=6 \mathrm{~m}, y=5 \mathrm{~m}$ for the receiver. Infinitesimally short dipoles or point sources are used. In the following figures, the received power is computed every $4 \mathrm{~cm}$ along the tunnel axis, i.e., from $z=1 \mathrm{~m}$ to $z=400 \mathrm{~m}$. In Fig. 1, one 2.4-GHz source, which is also noted as access point (AP), is located at $z=0 \mathrm{~m}$. This location could be right at a station.

In Fig. 2, the AP is now located on the opposite side, i.e., at $x_{0}=1 \mathrm{~m}, y_{0}=5.5 \mathrm{~m}$, and $z=400 \mathrm{~m}$. For example, this second location could be at the next station. The receiver moves along the same trajectory. The model reveals some important propagation characteristics of the radio propagation channel in the tunnel. Considering a narrow-band communication system, as reported by many authors [14], [15], we notice that the multipath causes small-scale fading and some sporadic severe attenuation of the signals. Energy also seems to fill the entire empty tunnel without the possibility of selectively addressing one or the other parallel track. To prevent cochannel interference, since the mean longitudinal attenuation remains low, consecutive APs need to work on distinct channels.

Suppose that a train is stopped along the track, at exactly the location of a severe attenuation. Then, the narrow-band communication could become locally interrupted, and the train would not be able to receive a data-enabling operation. Techniques like 


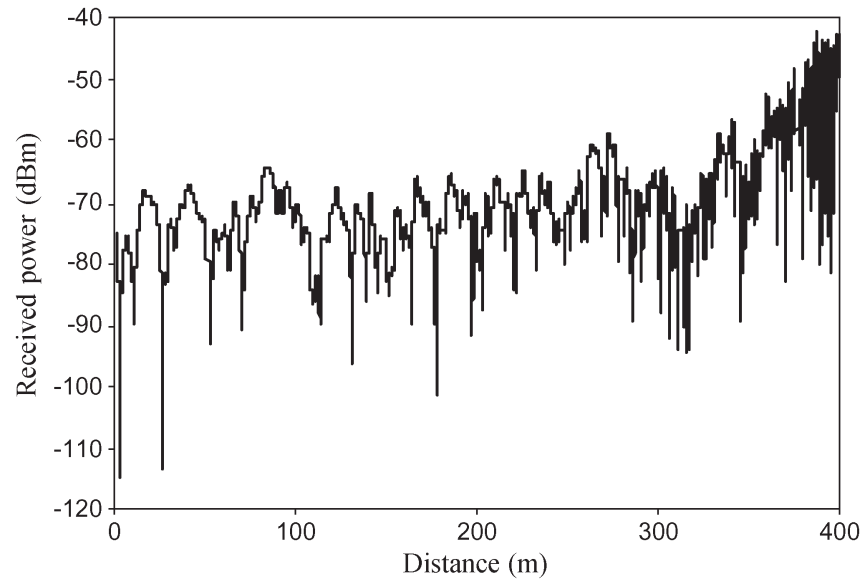

Fig. 2. Simulated second AP separated $400 \mathrm{~m}$ along the track.

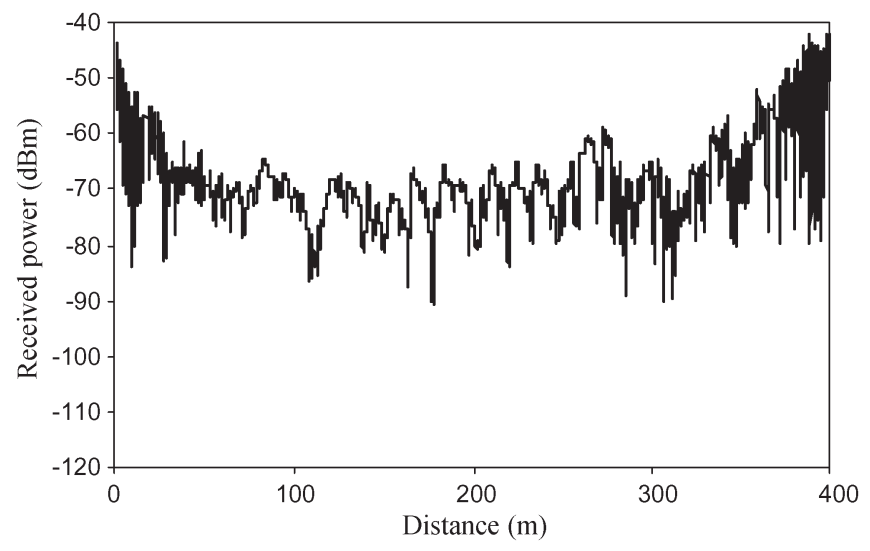

Fig. 3. Simulation of two transmitting APs separated $400 \mathrm{~m}$ apart.

space diversity, spread spectrum, or multiple input-multiple output (MIMO) are usually used to cope with this problem. Using MIMO techniques, Lienard et al. examined the possibilities of increasing the channel capacities in subway tunnels [16]. In tunnels, train antennas are generally installed at both extremity cabs to prevent the obstruction of the radio path to the next AP by the train's own structure. The best communication channel with the next front or back AP is then dynamically decided by the system. To illustrate this, and as an example of the space diversity scheme, we consider the two preceding APs situated at $z=0 \mathrm{~m}$ and $z=400 \mathrm{~m}$ simultaneously turned on at $2.4 \mathrm{GHz}$. Fig. 3 shows, for a receiving antenna situated at $x=6 \mathrm{~m}$, $y=5 \mathrm{~m}$, the strongest received power coming from either AP1 or AP2 as a function of the distance between the two APs.

Using this simple space diversity scheme, a reduced overall dynamic is obtained. As previously stated, a supplementary frequency diversity benefit would also be obtained from the two different consecutive AP-used channels. This would depend on the coherence bandwidth of the tunnel propagation channel and of the locally allocated channels. Since we use that straight tunnel model, we may consider that these results represent an ideal natural propagation situation, not taking into account supplementary attenuations and fading due to curves, bends, and branches, which were studied by Hwang et al. [17], but severe obstructions created by other moving trains located along the path to the communicating AP as well.

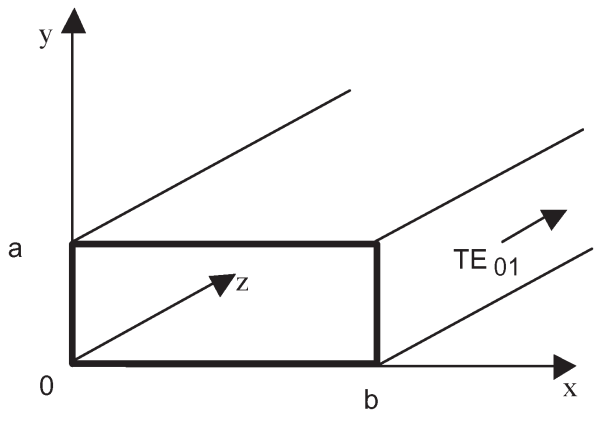

Fig. 4. Used waveguide geometry.

In the following sections, this ideal straight tunnel natural propagation case will be used as a base situation to be compared with the leaky waveguide solution.

\section{LEAKy WaVEguide Model}

Because it is easy to install and maintain along the track, a rectangular-shaped air-filled waveguide is considered. At $2.4 \mathrm{GHz}$, an aluminum rectangular waveguide of dimensions close to the Electronic Industries Alliance Waveguide WR 430 is used. The WR 430 internal dimensions are $10.92 \mathrm{~cm}$ wide by $5.46 \mathrm{~cm}$ high. These overall dimensions are similar to the dimensions used by the two-wire inductive-loop subway transmission lines often located in a plastic-coated wrapping laid along the line. These two-wire inductive-loop subway transmission lines are operated at much lower frequencies $(100 \mathrm{kHz})$ and offer limited bandwidth communications. Fig. 4 illustrates such a waveguide. Between 1.7 and $2.6 \mathrm{GHz}$, the waveguide is operated in its fundamental $\mathrm{TE}_{\mathrm{O} 1}$ mode.

The width-to-height ratio $b / a$ is usually set to 2 to maximize the usable bandwidth. In these conditions, the waveguide cutoff wavelength $\lambda_{c}$ is given by $\lambda_{c}=2 b$, and the relationship between $\lambda$, the wavelength in free space $\lambda_{c}$, this cutoff frequency, and the guided wavelength $\lambda_{g}$ is expressed as

$$
\left(\frac{1}{\lambda}\right)^{2}=\left(\frac{1}{\lambda_{c}}\right)^{2}+\left(\frac{1}{\lambda_{g}}\right)^{2}
$$

With regard to the group delay distortion, to achieve sufficient performance using channels up to $20 \mathrm{MHz}$ wide, frequencies close to the cutoff of the second $\mathrm{TE}_{\mathrm{O} 2}$ propagating mode $(2.6 \mathrm{GHz})$ are used [18]. In practice, the used communication channels could be WLAN channels according to the IEEE $802.11 \mathrm{~g}$ standard operated in the $2.4-\mathrm{GHz}$ band. The attenuation along such an air-filled waveguide is given by

$\alpha$ (in decibels per meter)

$$
=\frac{20}{\ln (10)} \frac{1}{b^{\frac{3}{2}}} \sqrt{2 \pi \varepsilon_{0} c \rho_{w g}} \frac{\left(\frac{f_{c}}{f}\right)^{\frac{3}{2}}+\left(\frac{b}{2 a}\right)\left(\frac{f}{f_{c}}\right)^{\frac{1}{2}}}{\left[1-\left(\frac{f_{c}}{f}\right)^{2}\right]^{\frac{1}{2}}}
$$

where $\varepsilon_{0}$ is the air dielectric constant, $c$ is the speed of light in free space, $\rho_{w g}$ is the resistivity of the waveguide metal, and $f$ and $f_{c}$ are, respectively, the operating frequency and the cutoff frequency, as deduced from (3). At $2.4 \mathrm{GHz}$, using an aluminum alloy that is mechanically compatible with the subway tunnel 


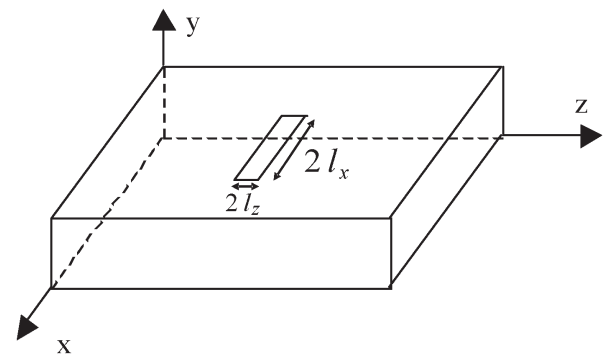

Fig. 5. Elementary slot perforation on a waveguide broad side (not to scale).

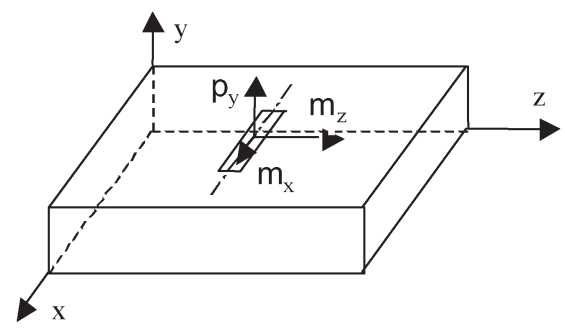

Fig. 6. Slot equivalent elementary dipoles.

environment, (4) provides a reasonable longitudinal attenuation that is close to $13 \mathrm{~dB} / \mathrm{km}$. To enable the coupling between the inside and outside of the waveguide, it is perforated along a broad side with thin short slots $(3 \mathrm{~mm}$ wide and less than $2 \mathrm{~cm}$ long). Fig. 5 shows such an elementary slot perforation on the rectangular-shaped waveguide. The slot is $2 l_{x}$ long by $2 l_{z}$ wide.

To study the problem of radiation of this small slot, Bethe's small-hole theory is used [19]. Using this theory, we consider that the radiation of this elementary slot is mainly equivalent to the radiation of equivalent dipoles. This equivalence is valid if the dimensions of the slots are short in relation to the wavelength if we consider a perfectly conductive infinite thin plane and if we consider radiation far from the aperture in terms of wavelengths [20]. In our case, these conditions are not strictly respected, since the aluminum waveguide thickness is on the order of $1 \mathrm{~mm}$, and the infinite plane is limited because of a waveguide width that is approximately five times the slot's greatest dimension [21]. However, the experimental results will show good accordance with this model [22]. Moreover, this model will then enable the following quick computation of the radiation of a very large set of slots. Thus, computing the radiation from this single slot can be reduced to the computation of the radiation of elementary dipoles, as shown in Fig. 6.

$p_{y}$ represents the electric equivalent dipole corresponding to the propagation of the illuminated $\mathrm{E}$ field normal component through the slot. $m_{x}$ and $m_{z}$ represent the magnetic dipoles created by the presence of the slot, interrupting $\mathrm{TE}_{\mathrm{O} 1}$ current lines inside the broad side of the waveguide.

Using Fig. 7 spherical coordinates, the radiation of an elementary magnetic dipole can be written as

$$
\begin{aligned}
H_{r} & =\frac{|\vec{m}|}{4 \pi} \frac{2 \cos \theta}{r^{3}}(1+j k r) e^{-j k r} \\
H_{\theta} & =\frac{|\vec{m}|}{4 \pi} \frac{\sin \theta}{r^{3}}\left(1+j k r-k^{2} r^{2}\right) e^{-j k r} \\
E_{\varphi} & =-j \omega \mu \frac{|\vec{m}|}{4 \pi} \frac{\sin \theta}{r^{2}}(1+j k r) e^{-j k r}
\end{aligned}
$$

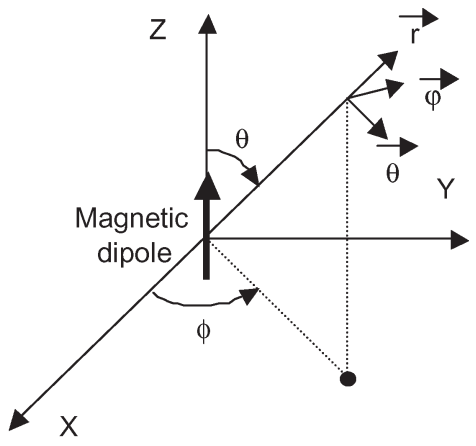

Fig. 7. Magnetic dipole system of coordinates.

where $H$ and $E$ are, respectively, the magnetic and electric field components, $k=(2 \pi / \lambda), \omega$ is the pulsation, and $\mu$ is the magnetic permeability. Using (7), a Cartesian coordinate system, and the particular slot orientation of Fig. 6, we obtain that $\vec{m}_{z}$ essentially generates an electric field component along the $x$-axis, and that $\vec{m}_{x}$ essentially generates an electric field component along the $z$-axis. In these expressions, the magnetic dipoles that are also expressed as

$$
\vec{m}=\psi_{m}: \vec{H}_{0}
$$

are a function of the illuminating $\vec{H}_{0}$ field inside the waveguide, and $\psi_{m}$ is the polarizability of the slot. For the $\mathrm{TE}_{\mathrm{O} 1}$ mode rectangular waveguide, we obtain the following relation between $H_{0}$ and the power $P$ inside the waveguide [23]:

$$
P=\frac{1}{4} H_{0}^{2} \pi^{2} \frac{a}{b} \frac{\lambda_{c}^{4}}{\lambda_{g}} \omega \mu .
$$

Using the same $2 l_{x}$ and $2 l_{z}$ slot cross dimensions, thin rectangular slot polarizabilities can be approximated by elliptical slot polarizabilities that have an analytical solution [24]. Along the long axis $2 l_{x}$ of an elliptical shape slot, the polarizability $\psi_{m x}$ is given by

$$
\psi_{m x}=\frac{2 \pi}{3} \frac{l_{x}^{3} e c c^{2}}{E(e c c)-K(e c c)}
$$

where ecc is the eccentricity of the ellipse, and $K(e c c)$ and $E(e c c)$ are, respectively, the complete elliptic integrals of the first and second kind. Computing the electric and magnetic polarizabilities of our very asymmetrical short slots, we obtain that $\psi_{m x}$ is more than ten times greater than $\psi_{m z}$ or the electric polarizability $\psi_{e}$. Thus, we will neglect these two last equivalent dipoles and consider that an electric field component oriented along the longitudinal axis of the leaky waveguide will mainly be radiated by the slot. To receive this electric field component, a cavity-backed rectangular slot is often used for the train antenna [25]. It has a broad radiation pattern along the waveguide axis. This characteristic is used to receive energy from several consecutive slots. It also has a good front-to-back ratio to attenuate signals reflected from the tunnel and the train environments. The train-board communication antenna moves along the track, approximately $20 \mathrm{~cm}$ over the waveguide. It receives this longitudinal electric field component. Other aperture geometries like circular holes would radiate more 


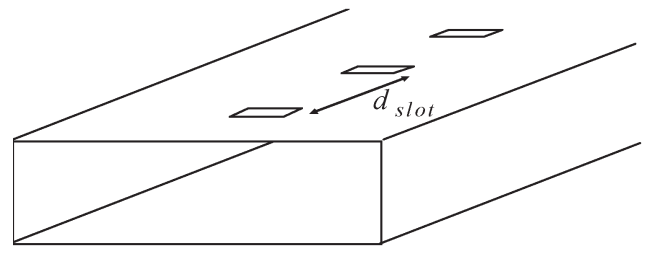

Fig. 8. Set of three regularly spaced slots along a section of the waveguide.

significant electric field components that would not be used by the train antenna but would extract more power from inside the waveguide. Thus, the slot geometry reduces the power extracted from the waveguide. Using (7)-(10), it is now possible to calculate the electric field radiated by a single slot perforated on the waveguide. Fig. 8 now represents a set of three regularly spaced slots that are perforated along a section of the waveguide.

Since these slots are very short compared with the wavelength, and since they are spaced at more than three times their longest dimension, we consider that the power radiated by each slot is very low, and that a low interaction exists between consecutive slots. Consequently, the power level transmitted along several meters of the waveguide is considered constant. Therefore, we may consider that the consecutive slot radiation can be represented by separate successive equivalent magnetic dipoles fed by the same power or existing illuminating magnetic field $H_{0}$ inside the waveguide. Considering that between two consecutive slots the illuminating magnetic field inside the waveguide is phase shifted by a factor $d_{\text {slot }} / \lambda_{g}$, and adding the contributions of each slot, it is now possible to calculate the electric field radiated by a long series of slots. The spacing $d_{\text {slot }}$ between the slots is adjusted so that, in the considered frequency range, moving along the waveguide, the total electric field remains almost constant over the waveguide, i.e., between two consecutive slots, over one slot. Between 2.4 and $2.5 \mathrm{GHz}$, this result was obtained using $d_{\text {slot }}=61 \mathrm{~mm}$.

The power radiated by a magnetic dipole is [23]

$$
P_{r m}=Z_{0} \frac{4}{3} \frac{\pi^{3}}{\lambda^{4}} m^{2}
$$

where $Z_{0}$ is the free-space wave impedance. This power is extracted by each consecutive slot from the waveguide, which results in an attenuation of the power inside the waveguide due to radiation. Considering the number of slots per unit length deduced from $d_{\text {slot }}$, it is possible to compute from (8)-(11) the total power taken from the waveguide per unit length. This longitudinal attenuation due to radiation is mainly tuned using the $l_{x}$ parameter. This additional longitudinal attenuation was set to $3 \mathrm{~dB} / \mathrm{km}$. This result was obtained using $2 l_{x}=19 \mathrm{~mm}$. $l_{z}$ is set for the slots to remain narrow but mechanically easily punched on the waveguide. $2 l_{z}$ was set to $3 \mathrm{~mm}$. Thus, at $2.4 \mathrm{GHz}$, we obtain a leaky waveguide that provides a longitudinal total attenuation of $16 \mathrm{~dB} / \mathrm{km}$, i.e., $13 \mathrm{~dB} / \mathrm{km}$ due to internal waveguide loss (4) plus $3 \mathrm{~dB}$ of supplementary attenuation due to radiation. This verifies our preceding assumption considering that along short waveguide sections an almost constant power is traveling inside the waveguide.

On-site experiments consistently show values of $17 \mathrm{~dB} / \mathrm{km}$ on up to $1.5-\mathrm{km}$-long waveguide installations. The $1-\mathrm{dB} / \mathrm{km}$ difference was attributed to the supplementary attenuation due

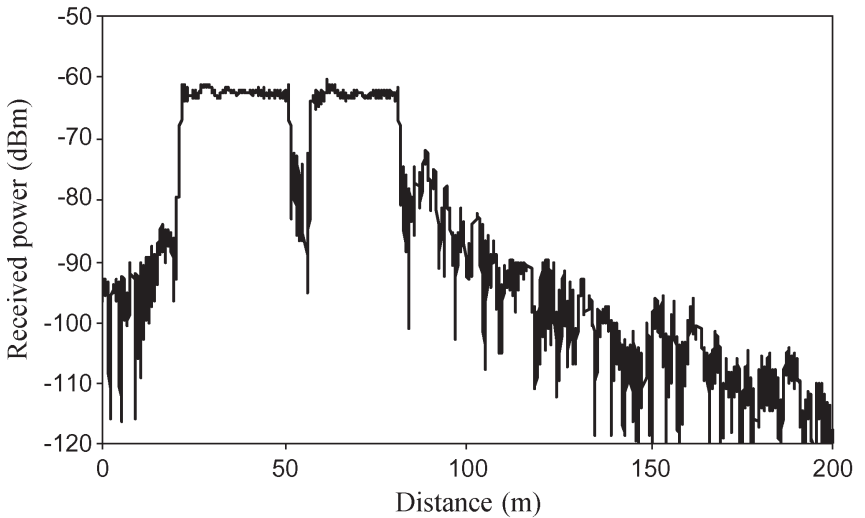

Fig. 9. Radiation of two closely spaced consecutive lengths of the leaky waveguide in a tunnel.

to waveguide-connecting flanges, which are located every $15 \mathrm{~m}$. This figure is much better than the $80 \mathrm{~dB} / \mathrm{km}$ provided by leaky coaxial cables and remains competitive with the $15-\mathrm{dB} / \mathrm{km}$ natural propagation average attenuation that is obtained at $2.45 \mathrm{GHz}$ by Lienard et al. in very long railway tunnels [26].

Using these assumptions, each of these elementary slots located along the waveguide can now be considered as a separate source radiating in the tunnel, with the two consecutive sources being separated along the tunnel axis by $d_{\text {slot }}$. Then, we can reuse the preceding ray-optical modeling of the radio propagation provided in [12] to compute this long series of slot radiation in the tunnel.

\section{Tunnel Radio Coverage Simulation Using LEAKY WAVEGUIDES}

Using this model, and for the preceding base case using a single transmitting source, we consider an 8-m-wide and 6-m-high infinite empty rectangular-shaped tunnel. A source of $0 \mathrm{dBm}$ at $2.4 \mathrm{GHz}$ is injected into the leaky waveguide sections. The leaky waveguide sections are laid along the tunnel $z$-axis at $6 \mathrm{~m}$, $0.3 \mathrm{~m}$ (width, height), i.e., the horizontal waveguide slots are situated $30 \mathrm{~cm}$ above the ground. A source is considered every $d_{\text {slot }}$. An infinitesimally short dipole or point source is used for receiving. Considering the cavity-backed rectangular slot often used for the train antenna, a point source receiving antenna will not attenuate the reflected signals coming from some of the tunnel walls, thus providing a good way to evaluate the effective impact of the surrounding tunnel on the communication. The received power is computed along the $z$-axis at $x=6 \mathrm{~m}$ and $y=0.6 \mathrm{~m}$, i.e., $30 \mathrm{~cm}$ above the waveguide slot plane. Figs. 9 and 10 show two different simulation configurations and the associated results. In Fig. 9, a first 30-m-long waveguide section is laid between $z=20 \mathrm{~m}$ and $z=50 \mathrm{~m}$; it is then interrupted for $5 \mathrm{~m}$, and then, a second 25-m-long leaky waveguide section is laid between $z=55 \mathrm{~m}$ and $z=80 \mathrm{~m}$. The phase of the signal injected into the second section corresponds to the phase of the signal as if it continues to propagate into the waveguide along the 5-m-long interruption.

Analyzing the radiated signal and starting from $z=0 \mathrm{~m}$, we first obtain a residual signal propagated into the tunnel that is coming from the far radiation of the array of slots. Along 


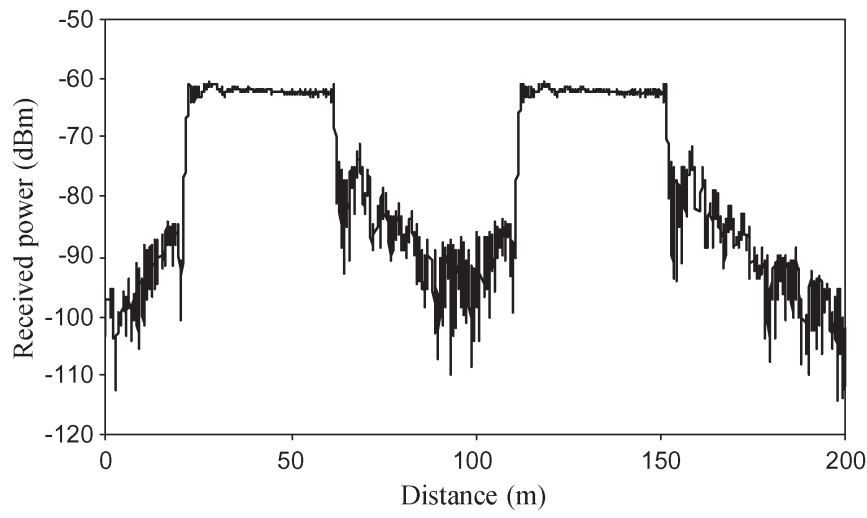

Fig. 10. Radiation of two limited lengths of the leaky waveguide in a tunnel.

this waveguideless section, the fast fading is associated with this signal. At $z=20 \mathrm{~m}$, as soon as the receiving position is located right over the very first slots of the waveguide, the received power increases very quickly by about $20 \mathrm{~dB}$; then, it remains very stable all along the first leaky waveguide section and not significantly affected by tunnel propagation. After the end of the first waveguide section, the received power decreases very quickly to increase very abruptly for some $20 \mathrm{~dB}$ over the second section. Leaving the second leaky waveguide section at $z=80 \mathrm{~m}$, a residual signal propagated into the tunnel and affected by fading slowly decreases down to our selected 200-m computation range.

Fig. 10 shows the results obtained using a slightly different configuration. Both leaky waveguides are $40 \mathrm{~m}$ long and situated between $z=20 \mathrm{~m}$ and $z=60 \mathrm{~m}$ for the first section and $z=110 \mathrm{~m}$ to $z=150 \mathrm{~m}$ for the second section, respectively. The phase of the signal injected into the second section corresponds to the phase of the signal as if it continues to propagate into the waveguide along the 50-m-long interruption. Since a wider distance is considered between the two leaky waveguide sections, a better decoupling is obtained between the following sections. Comparing Figs. 9 and 10, it is easier to detect on the latter that the signal increase at $z=20 \mathrm{~m}$ or $z=110 \mathrm{~m}$ is steeper than the signal decrease at $z=60 \mathrm{~m}$ and $z=150 \mathrm{~m}$. This is attributed to the directivity of the endfed very long array of slots. This directivity is focused from the transmitter extremity at $z=20 \mathrm{~m}$ and $z=110 \mathrm{~m}$ to the extremity of the array at $z=60 \mathrm{~m}$ and $z=150 \mathrm{~m}$. On a 40-m-long leaky waveguide section, an array of more than 600 slots is considered.

Since the radio-frequency energy is carried in the waveguide nearly up to the train antenna, and the outside path to the antenna is short, we deduce from Figs. 9 and 10 that, over the leaky waveguide, radio propagation becomes almost independent of the tunnel environment. Adequately adjusting the slots and waveguide parameters, we obtain a practically constant received power over the waveguide that is progressively attenuated along the track. Using a set of optimized parameters for the slot dimensions and spacing, the simulations were also performed at $2.5 \mathrm{GHz}$, which provides similar results over this $100-\mathrm{MHz}$ frequency range.

In Fig. 3, when introducing the base antenna coverage case, we simulated the case of two consecutive APs $400 \mathrm{~m}$ apart.
Since a low longitudinal attenuation was observed, we deduced that two different channels were needed to limit the cochannel interference along the path. However, in Fig. 3, looking at the high fading between the two APs, we also deduce that effectively managing the handover between the two APs is not so easy. From Figs. 9 and 10, we notice that the handover management could more easily be performed between two consecutive APs connected to separate consecutive lengths of leaky waveguides. Since the attenuation from one waveguide to the following is significant, the same channel could also probably be used. This will lessen the frequency spectrum CBTC requirements.

In subway tunnels, the train size is close to the tunnel size, which leaves little space for radio propagation by use of natural propagation when another train is inserted between the AP and the train communicating with this AP. During subway exploitation, several trains can be present in the same interstation tunnel between two consecutive APs. This creates a heavy and potentially train-long obstruction of the radio path between a communicating train and the next AP [27], [28]. This obstruction is dependant on the train structures and on the relative train locations along the tunnel. Consequently, it would be a very hard task to accurately and dynamically simulate all these obstruction scenarios, and it would also be very difficult to demonstrate the operator's stringent requirement of a $100 \%$ available CBTC anytime and anywhere along the track. In these critical areas, a leaky waveguide system could be installed, to be independent of these poor and uncertain propagation conditions. Thus, a mixed solution, using leaky waveguides at critical locations and antennas in more predictable environments, can be an effective solution. This mixed system requires separate train-board antennas to communicate through the waveguide and using natural propagation. Considering the waveguide typical installation on the track (Section VII and Fig. 12), a first train antenna has to laterally be installed on the cab, being mechanically maintained at a short distance over the waveguide $(20-30 \mathrm{~cm})$. For natural propagation, two other train-board antennas have to be installed at both extremity cabs of the train. They are generally located in the upper front of the cab to prevent the obstruction of the radio path to the next AP by the train's own structure. To illustrate this mixed solution, we started with the same leaky waveguide disposition as in Fig. 9 or Fig. 10. A limited length of the leaky waveguide is simulated along the tunnel from $z=25 \mathrm{~m}$ to $z=100 \mathrm{~m}$. An AP is also simulated at $x=6 \mathrm{~m}, y=5 \mathrm{~m}$, and $z=400 \mathrm{~m}$. Since we consider an empty tunnel and no particular antenna characteristic, we do not move the tunnel cross-section receiving location going from the waveguide to the natural propagation. Thus, the receiving antenna location is always $6 \mathrm{~m}, 0.3 \mathrm{~m}$ (width, height). In Fig. 11, the computed received power is shown along the 400-m path.

The radiation characteristics of the two technical solutions can be recognized in Fig. 11. Between $z=25 \mathrm{~m}$ and $z=$ $100 \mathrm{~m}$, an almost constant received power over the leaky waveguide is obtained. When over the leaky waveguide, there is little effect related to tunnel propagation. The tunnel natural propagation characteristics reappear very quickly after $z=100 \mathrm{~m}$. 


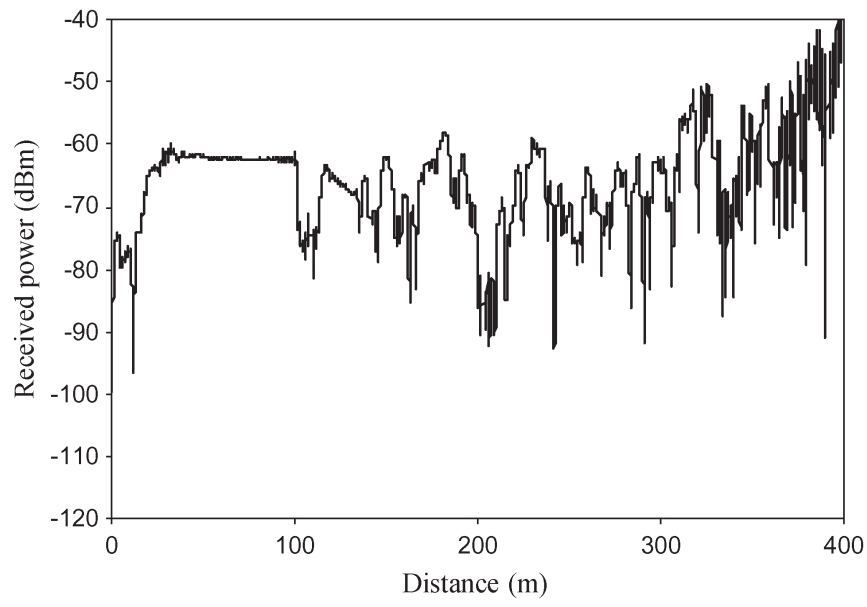

Fig. 11. Radiation of a limited length of the leaky waveguide and an antenna in a tunnel.

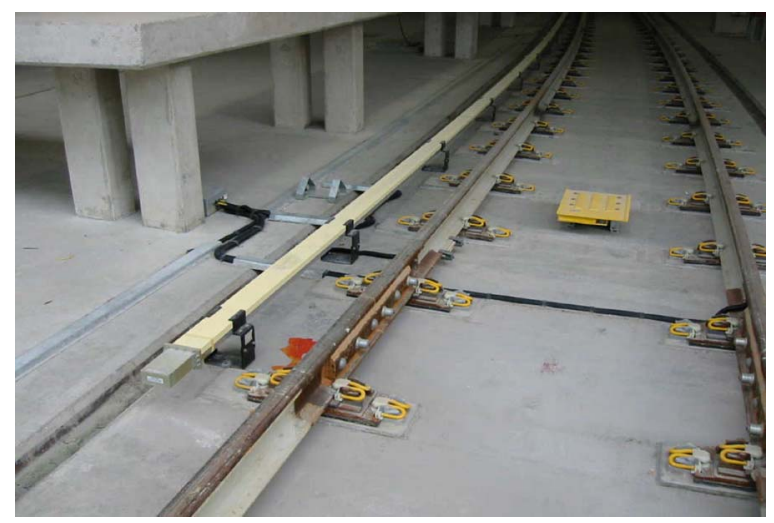

Fig. 12. Typical leaky waveguide installation along a subway track.

\section{EXAMPLE OF LEAKY WAVEGUIDE IMPLEMENTATION}

Of course, when it comes to the real radio coverage between stations, much longer sections of the leaky waveguide are actually used. Sections that are up to $1 \mathrm{~km}$ long have been used. Fig. 12 shows an operational configuration.

The leaky waveguide is laterally installed on the left side of the track of a recently opened line of a rapid transit system. The train-board communication antenna moves along the track (approximately $20 \mathrm{~cm}$ over the waveguide). To prevent moisture from penetrating the structure, the leaky waveguide is covered by a low smoke emission dielectric radome. The dielectric radome that is covering and protecting the slots increases their equivalent electric length. This is compensated by slightly diminishing the mechanical length $2 l_{x}$ of each slot [29]. The extremities of the waveguide sections are terminated either on a waveguide-to-coaxial transition or on an adapted load. On the track, between the two rails, a localization beacon can also be seen, which is used for the high-accuracy halting of trains at station gates.

Fig. 13 shows another leaky waveguide installation used this time along two parallel tracks. One leaky waveguide is laterally installed along each track. Waveguides are alternatively installed on each side of the track, depending on the local mechanical track constraints. At the end of each waveguide section, waveguide-to-coaxial transitions, which are associated

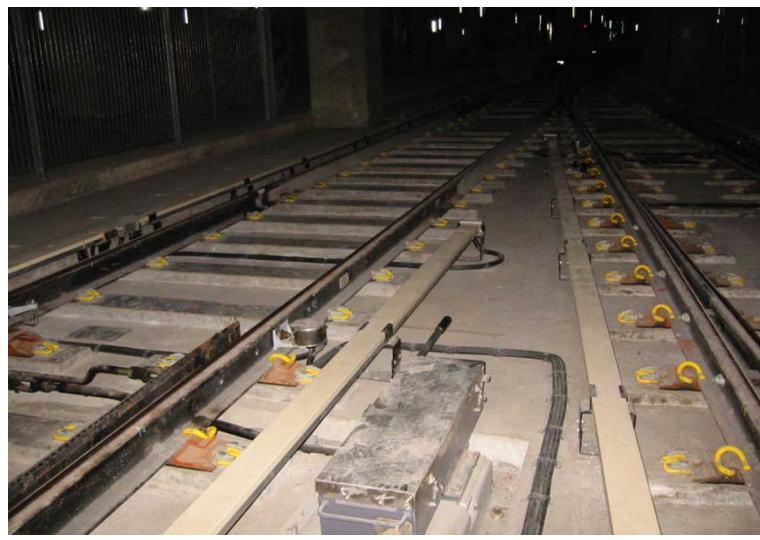

Fig. 13. Typical installation of the leaky waveguide in a parallel-track configuration.

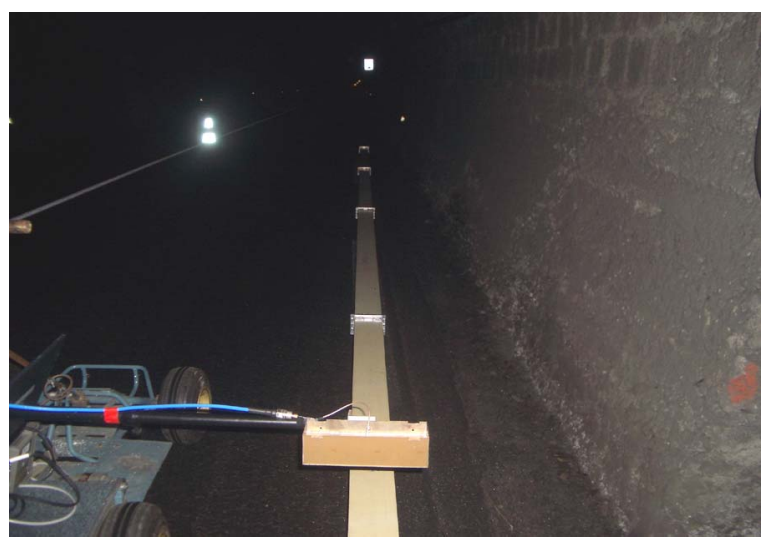

Fig. 14. Leaky waveguide laterally installed along the tunnel and the receiving antenna moving along.

with short sections of low-loss coaxial cable, are used. These transitions are used to connect the leaky waveguide section to the AP radio modems.

\section{Tunnel ExPERImental Measurements}

The experimental measurements were carried out in a straight 3300-m-long empty tunnel. A 25-m-long leaky waveguide is installed in the deep part of this tunnel at approximately $30 \mathrm{~cm}$ above the ground and $1 \mathrm{~m}$ from the wall. It is fed by a $10-\mathrm{dBm} 2.4-\mathrm{GHz}$ source. Fig. 14 shows this experimental setup.

As often used for the train-board antenna, a cavity-backed rectangular slot is used for the receiving antenna. The reference gain of the air rectangular cavity slot antenna is $5.8 \mathrm{dBi}$ [25]. It has a broad front radiation pattern and a measured front-toback ratio that is better than $18 \mathrm{~dB}$ [30]. The leaky waveguide slots and the receiving antenna slot are positioned face to face. They use the same orientation to maintain the right polarization. During the measurements, a lorry runs very slowly, parallel to the waveguide. Moving along, the lorry maintains the receiving antenna at a constant height, vertically above the leaky waveguide. The antenna signal is measured and recorded every $4 \mathrm{~cm}$ using a spectrum analyzer and a PC embarked on the lorry. Figs. 15-17 illustrate the results obtained with the receiving 


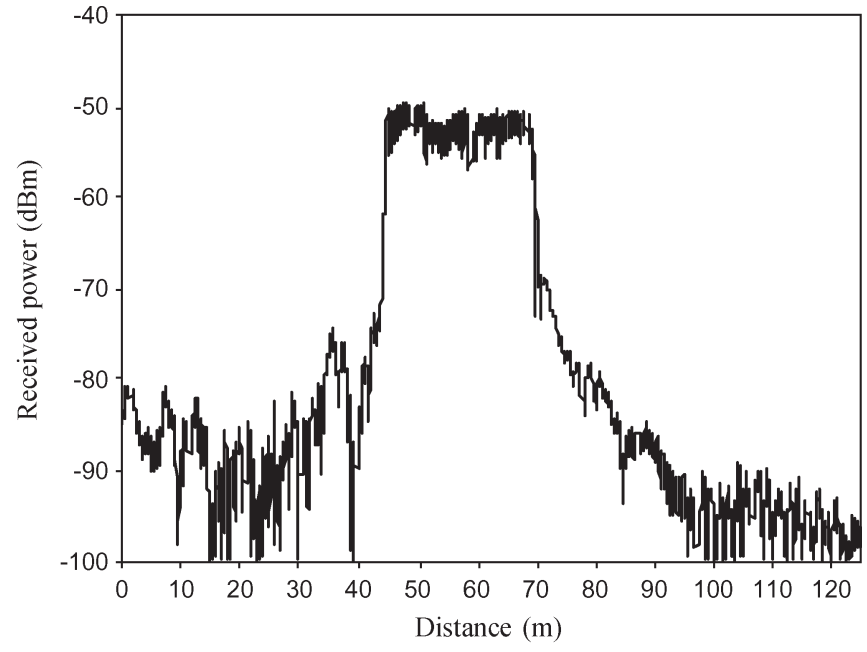

Fig. 15. Received power at $20 \mathrm{~cm}$ above the leaky waveguide.

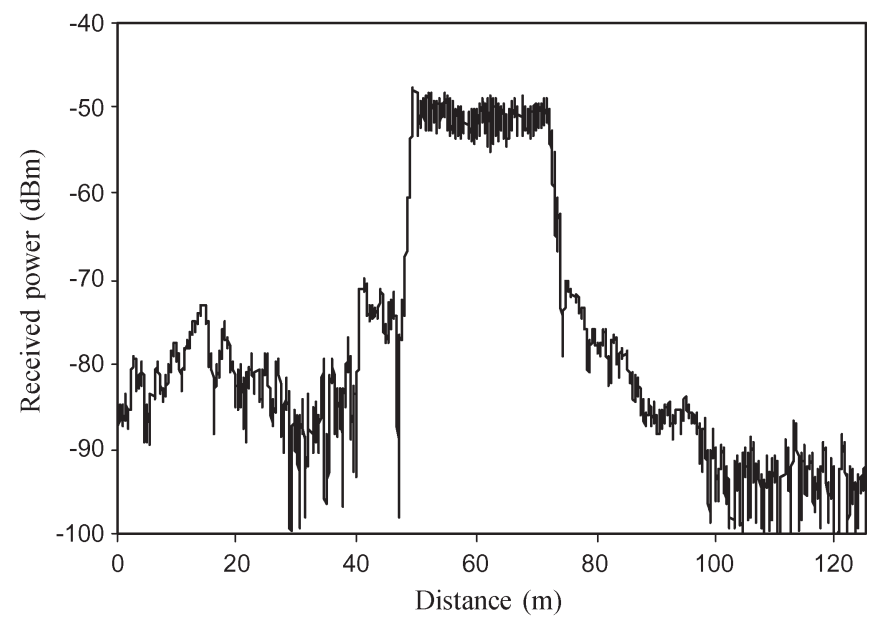

Fig. 16. Received power at $30 \mathrm{~cm}$ above the leaky waveguide.

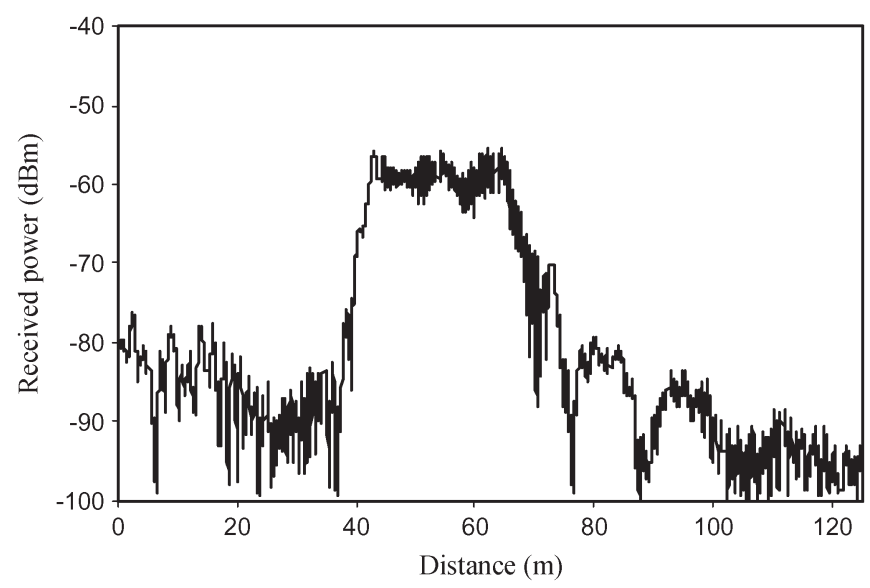

Fig. 17. Received power at $80 \mathrm{~cm}$ above the leaky waveguide.

antenna moving at 20,30 , and $80 \mathrm{~cm}$, respectively, over the waveguide.

At the limited 20- and 30- $\mathrm{cm}$ heights over the waveguide, Figs. 15 and 16 exhibit similar experimental results. A 62-dB coupling loss is measured. The coupling loss represents the

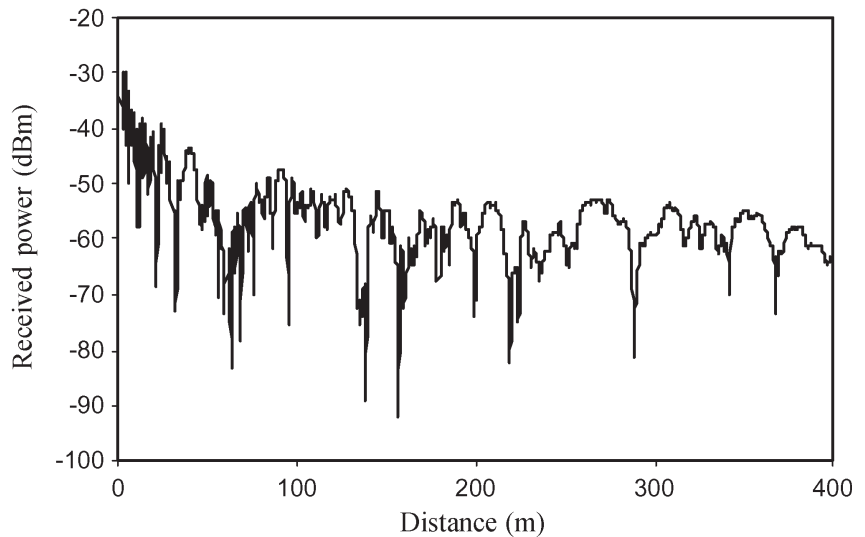

Fig. 18. Received power from the antenna.

ratio between the power propagating in the waveguide and the train antenna received power [31].

Similar conclusions to the simulation results provided in Figs. 9 and 10 can be deduced. When over the leaky waveguide, there is little effect related to tunnel propagation. This is probably enhanced by the good front-to-back ratio of the used receiving antenna, attenuating some of the signals reflected from the tunnel walls. In Fig. 17, at $80 \mathrm{~cm}$ over the waveguide, the conditions change a lot; an 8-dB loss of signal is obtained, and the tunnel propagation conditions seem to significantly impact on the received signal. The received power increases slower after the first slots. The end of the waveguide becomes more difficult to locate.

To conclude this comparison, Fig. 18 shows the results obtained using natural propagation in this particular tunnel. Two of the previous cavity-backed rectangular slot antennas were used for emitting and receiving $2.4-\mathrm{GHz}$ signals. The transmitting antenna, which is fed by a power of $10 \mathrm{dBm}$, is located on a pole at $3 \mathrm{~m}$ above the ground and 1 from the lateral wall. We used vertical polarization. On the lorry, the receiving antenna used the same setup that was previously used, but the receiving antenna was turned $90^{\circ}$ along its axis for the slot plane to vertically be directed, pointed at the emitting antenna. As stated before, in real operations, the natural propagation antennas are generally located in the upper front of the cabs to prevent the obstruction of the radio path to the next AP by the train's own structure. Since our experimentations use an empty tunnel, this particular lorry receiving antenna location was considered adequate. The received signals are measured along a 400-m-long path. Fig. 18 shows the received power along this path.

The previously mentioned characteristics of natural propagation in the tunnel apply to these results.

\section{CONCLUSION}

The current requirements for CBTC, particularly for automated subway systems, necessitate significantly high data rates, i.e., on the order of $10 \mathrm{Mb} / \mathrm{s}$. Communication ranges of several hundred meters between stations are also required. To achieve these high transmission rates, the current technical solutions use radio modems that work at high carrier frequencies (between 2 
and $6 \mathrm{GHz}$ ). In this paper, a natural propagation link using antennas and a leaky waveguide antenna alternative solution have been compared. Although the antenna solution is very simple and effective, it was demonstrated that the leaky waveguide solution yields to some improvements when it comes to predicting the radio coverage, managing handovers between consecutive APs, or to reducing the need for an allocated radio-frequency spectrum. These significant advantages have to be balanced with operator concerns about the necessity of putting additional equipment at track level or at the tunnel ceiling. To limit this impact, the propagation line has been built very robust, empty, and easy to assemble and disassemble. Upgrading the leaky waveguide system between 5 and $6 \mathrm{GHz}$ will still produce an effective solution and would significantly reduce the size of the radiating structure.

\section{REFERENCES}

[1] E. R. Leech and W. T. Outlaw, "A communication system custom designed for the Montreal metro rapid transit subway," in Proc. Veh. Technol. Conf., Mar. 16-18, 1977, vol. 27, pp. 137-143.

[2] L. Zhi-Wei, "Network OAM requirements for the New York City transit network," IEEE Commun. Mag., vol. 42, no. 10, pp. 112-116, Oct. 2004.

[3] D. B. Seidel and J. R. Wait, "Transmission modes in a braided coaxial cable and coupling to a tunnel environment," IEEE Trans. Microw. Theory Tech., vol. MTT-26, no. 7, pp. 494-499, Jul. 1978.

[4] P. Delogne and L. Deryck, "Underground use of a coaxial cable with leaky sections," IEEE Trans. Antennas Propag., vol. AP-28, no. 6, pp. 875-883, Nov. 1980.

[5] T. Suzuki, T. Hanazawa, and S. Kozono, "Design of a tunnel relay system with a leaky coaxial cable in an $800-\mathrm{MHz}$ band land mobile telephone system," IEEE Trans. Veh. Technol., vol. VT-29, no. 3, pp. 305-316, Aug. 1980.

[6] J. H. Wang and K. K. Mei, "Theory and analysis of leaky coaxial cables with periodic slots," IEEE Trans. Antennas Propag., vol. 49, no. 12, pp. 1723-1732, Dec. 2001.

[7] K. Mikoshiba and Y. Nurita, "Guided radiation by coaxial cable for train wireless systems in tunnels," IEEE Trans. Veh. Technol., vol. 18, no. 2, pp. 66-69, Aug. 1969

[8] T. Kawakami, T. Maruhama, T. Takeya, and S. Kohno, "Waveguide communication system for centralized railway traffic control," IRE Trans. Veh. Commun., vol. VC-13, no. 1, pp. 1-18, Sep. 1959.

[9] D. Didascalou, T. M. Schafer, F. Weinmann, and W. Wiesbeck, "Raydensity normalization for ray-optical wave propagation modeling in arbitrarily shaped tunnels," IEEE Trans. Antennas Propag., vol. 48, no. 9, pp. 1316-1325, Sep. 2000.

[10] D. Didascalou, J. Maurer, and W. Wiesbeck, "Subway tunnel guided electromagnetic wave propagation at mobile communications frequencies," IEEE Trans. Antennas Propag., vol. 49, no. 11, pp. 1590-1596, Nov. 2001.

[11] S. F. Mahmoud and J. R. Wait, "Geometrical optical approach for electromagnetic wave propagation in rectangular mine tunnels," Radio Sci., vol. 9, no. 12, pp. 1147-1158, 1974.

[12] Y. P. Zhang and H. J. Hong, "Ray-optical modeling of simulcast radio propagation channels in tunnels," IEEE Trans. Veh. Technol., vol. 53, no. 6, pp. 1800-1808, Nov. 2004.

[13] Y. P. Zhang and Y. Hwang, "Theory of the radio-wave propagation in railway tunnels," IEEE Trans. Veh. Technol., vol. 47, no. 3, pp. 10271036, Aug. 1998.

[14] M. Lienard and P. Degauque, "Propagation in wide tunnels at $2 \mathrm{GHz}$ : A statistical analysis," IEEE Trans. Veh. Technol., vol. 47, no. 4, pp. 13221328, Nov. 1998.
[15] Y. P. Zhang, Y. Hwang, and R. G. Kouyoumjian, "Ray-optical prediction of radio-wave propagation characteristics in tunnel environments-Part 2: Analysis and measurements," IEEE Trans. Antennas Propag., vol. 46, no. 9, pp. 1337-1345, Sep. 1998.

[16] M. Lienard, P. Degauque, J. Baudet, and D. Degardin, "Investigation on MIMO channels in subway tunnels," IEEE J. Sel. Areas Commun., vol. 21, no. 3, pp. 332-339, Apr. 2003.

[17] Y. Hwang, Y. P. Zhang, and R. G. Kouyoumjian, "Ray-optical prediction of radio-wave propagation characteristics in tunnel environments-Part 1: Theory," IEEE Trans. Antennas Propag., vol. 46, no. 9, pp. 1328-1336, Sep. 1998.

[18] M. M. Brady, "Cutoff wavelengths and frequencies of standard rectangular waveguides," Electron. Lett., vol. 5, no. 17, pp. 410-412, Aug. 1969.

[19] H. A. Bethe, "Theory of diffraction by small holes," Phys. Rev., vol. 66, no. 7/8, pp. 163-182, Oct. 1944.

[20] J. Van Bladel, "Field penetration through small apertures: The first-order correction," Radio Sci., vol. 14, pp. 319-331, Mar./Apr. 1979.

[21] B. Radak and R. L. Gluckstern, "Penetration of electromagnetic fields through an elliptical hole in a wall of finite thickness," IEEE Trans. Microw. Theory Tech., vol. 43, no. 1, pp. 194-204, Jan. 1995.

[22] H. A. Mendez, "Shielding theory of enclosures with apertures," IEEE Trans. Electromagn. Compat., vol. EMC-20, no. 2, pp. 296-305, May 1978.

[23] R. E. Collin, Field Theory of Guided Waves. New York: McGraw-Hill, 1960.

[24] S. B. Cohn, "The electric polarization of apertures of arbitrary shapes," Proc. IRE, vol. 40, no. 9, p. 1069, 1952.

[25] R. C. Johnson and H. Jasik, Antenna Engineering Handbook. New York: McGraw-Hill, 1961, ch. 8.

[26] M. Lienard, P. Degauque, and P. Laly, "Long-range radar sensor for application in railway tunnels," IEEE Trans. Veh. Technol., vol. 53, no. 3, pp. 705-715, May 2004.

[27] H. D. Hettstedt, R. Harthun, M. Davies, P. Chan and E. Cheung, "The PCS service in the MTRC subway in Hong Kong," in Proc. ITC Conf. Hong Kong, Feb. 1999, p. 6. [Online]. Available: http://www. rfsworld.com/userfiles/pdf/pcs-service.pdf

[28] M. Lienard, P. Degauque, and P. Laly, "Communication and distance measurement in subway tunnels using natural propagation," in Proc. 5th IEEE Int. Conf. Devices, Circuits Syst., Caracas, Venezuela, Nov. 3-5, 2004, vol. 1, pp. 240-243.

[29] M. Bailey, "Design of dielectric-covered resonant slots in a rectangular waveguide," IEEE Trans. Antennas Propag., vol. AP-15, no. 5, pp. 594598, Sep. 1967.

[30] W. Hong, N. Behdada and K. Sarabandi, Miniaturized Cavity Backed Slot Antenna. Ann Harbor, MI: Dept. Elect. Eng. Comput. Sci., Univ. Michigan, 2005. [Online]. Available: http:// www.eecs.ucf.edu/ behdad/papers/Allerton_2005.pdf

[31] W. H. Tranter, T. S. Rappaport, B. D. Worerner, and J. H. Reed, Wireless Personal Communications Emerging Technologies for Enhanced Communications. Norwell, MA: Kluwer, 1999, ch. 2.

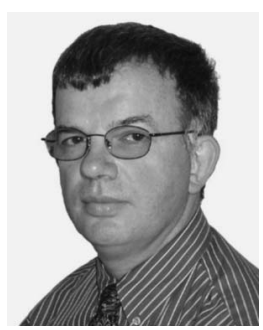

Marc Heddebaut (M'03) was born in Somain, France, in 1955. He received the M.S. and Ph.D. degrees in electronics from the University of Lille, Lille, France, in 1980 and 1983, respectively.

Since 1983, he has been with the French National Institute for Transportation and Safety Research (INRETS), Bron Cedex, France, where he became a Senior Researcher in 1988. Since 1979, he has been working in the field of land mobile communication and electromagnetic compatibility. His primary interests are telecommunication systems dedicated to land transport, mobile localization, and command control of automated vehicles. 